\title{
Initial Clinical Experience of Repeat Thrombectomy with a Retrieval Stent (RTRS) with Continuous Proximal Flow Arrest by Balloon Guide Catheter for Acute Intracranial Carotid Occlusion
}

\author{
Wen-huo Chen $\left(\mathbb{D}\right.$, Tingyu Yi $\mathbb{D}^{D}$, Yan-Min Wu $\mathbb{D}^{\text {, }}$, Zhi-nan Pan $\mathbb{D}^{\circ}$, Xiu-fen Zheng $\mathbb{D}$, \\ Xiao-hui Lin $\mathbb{D}$, Ding-lai Lin $\mathbb{D}$, and Rong-cheng Chen \\ Department of Neurointervention, Zhangzhou Affiliated Hospital of Fujian Medical University, Fujian, China \\ Correspondence should be addressed to Tingyu Yi; siyuyufen@163.com
}

Received 27 September 2021; Accepted 29 November 2021; Published 31 December 2021

Academic Editor: Muh-Shi Lin

Copyright (c) 2021 Wen-huo Chen et al. This is an open access article distributed under the Creative Commons Attribution License, which permits unrestricted use, distribution, and reproduction in any medium, provided the original work is properly cited.

\begin{abstract}
Background. Balloon guide catheters (BGCs) have good performance in terms of radiological outcomes in acute ischemic thrombectomy. It is not uncommon for BGCs to be blocked by thrombi, especially in cases with acute intracranial internal carotid artery (ICA) occlusion. Our initial experience using repeat thrombectomy with a retrieval stent (RTRS) with continuous proximal flow arrest by BGC for acute intracranial ICA occlusion is presented. Methods. In patients with acute intracranial ICA occlusion treated with RTRS, clinical data, including the National Institutes of Health Stroke Scale (NIHSS) score at admission and modified Rankin Scale (mRS) score at 90 days, and procedural data, including the Extended treatment in Cerebral Infarction (eTICI) score, procedural time, and complications, were analyzed. Results. Thirty-two consecutive patients (12 men (37.5\%); mean age: 73 years) were treated with RTRS using a BGC. The median NIHSS score was 19 . The median puncture-to-reperfusion time was 46 minutes (range: 22-142 minutes). All patients were successfully revascularized; eTICI $2 \mathrm{c}$ or better recanalization was achieved in 30 (93.8\%) patients. No procedure-related complications or symptomatic intracranial hemorrhage occurred. Two cases $(6.3 \%)$ had distal emboli, but none had emboli to the anterior cerebral artery. Fourteen patients (43.8\%) achieved a good outcome with an mRS score of 0-2 at 90 days, and 8 patients (25.0\%) died. Conclusions. In patients with intracranial ICA occlusion, RTRS with proximal flow arrest by BGC is effective and safe, achieving good clinical and angiographic outcomes. This method may reduce the incidence of distal emboli in thrombectomy with stent retrievers.
\end{abstract}

\section{Introduction}

Acute intracranial internal carotid artery (ICA) occlusion, especially carotid $\mathrm{T}$ occlusion, is often associated with a large clot burden, low clot burden score, poor collateral flow, and poor outcome, resulting in a large ICA territory infarction [1]. Its overall mortality is up to $51 \%$ [1]. Endovascular therapy is the standard treatment for acute large intracranial artery occlusion [2-8]. Despite major strides in reducing disability from large vessel occlusion strokes with stent retrievers (SRs), $72 \%$ of patients with intracranial ICA occlusion remain physically disabled [1]. A high first-pass complete reperfusion rate [9], short puncture-to-reperfusion time (PRT) [10], and low incidence of distal emboli [11] are associated with improved functional neurological recovery.

Balloon guide catheters (BGCs) offer reliable blood flow arrest and flow reversal in combination with aspiration via syringes or high-flow pump systems [12]. Nonrandomized studies suggest that BGC use during mechanical thrombectomy for acute ischemic stroke is associated with superior clinical and angiographic outcomes [13]. However, in the setting of acute intracranial ICA occlusion with a large clot burden, it is very possible that BGCs is blocked by thrombi. In the clinical setting, when the guide catheter is blocked by a thrombus, no blood flow comes out of the catheter, and the guide catheter will be retracted to clear the thrombus in it. 
However, such an operation wastes time and may increase the possibility of distal emboli occurrence, which may influence patients' clinical outcome.

The purpose of our study was to assess the feasibility of repeat thrombectomy with a retrieval stent (RTRS) with continuous proximal flow arrest by BGC and to evaluate whether the use of this technique improves reperfusion and clinical outcomes in acute intracranial ICA occlusion.

\section{Methods}

2.1. Study Patients. The study included patients identified from our prospective registry database of acute stroke patients treated with endovascular therapy between January 1, 2015, and November 30, 2020. The selected patients met the following criteria: (1) ischemic stroke resulting from acute intracranial ICA occlusion confirmed by digital subtraction angiography (DSA); (2) patient age greater than 18 years; (3) prestroke modified Rankin Scale (mRS) score of $0-1$; and (4) receiving endovascular therapy with the use of a BGC.

The exclusion criteria for this study were thrombectomies performed for (1) ipsilateral stenosis or acute extracranial occlusions, (2) previously deployed stents, and (3) dissections and in settings in which (4) the BGC was not blocked by a thrombus during the procedure and (5) the BGC was blocked by a thrombus but the RTRS technique was not used.

\subsection{Endovascular Procedure: RTRS Technique. The RTRS technique is shown in Figure 1.}

All procedures were performed under general anesthesia or conscious sedation. After femoral puncture with an $8 \mathrm{~F}$ sheath, 2500 units or less of intravenous (IV) heparin was injected. An 8F BGC (Merci/Flowgate 2, Stryker Products, Boston, USA) was advanced with a $4 \mathrm{~F} / 5 \mathrm{~F}$ MPA catheter or via the exchange technique and placed distal to the carotid bifurcation. After the presence of acute intracranial ICA occlusion was confirmed by injection of contrast through the BGC and in some cases a distal access catheter (Catalyst, Stryker Products, Boston, USA; Navein, ev3 Inc., Plymouth, MN, USA) was applied, a 0.014-inch microwire (Transcend; Stryker, Kalamazoo, MI) was passed through the intracranial ICA occluded segment, and a microcatheter (ev3 Inc., Plymouth, MN, USA) was advanced over the microwire through the occlusion site. Microcatheter injection was performed to verify the microcatheter's position distal to the thrombus; then, a Solitaire AB 6-30 mm (ev3 Inc., Plymouth, MN, USA) was unsheathed at the occlusion site. After at least 3 minutes, the balloon was inflated to arrest the anterograde flow from the ICA, and the fully deployed Solitaire stent was partially resheathed by microcatheter or distal access catheter. Together with the delivery microcatheter and distal access catheter if used, the stent was gently pulled back under continuous suction achieved with a syringe (Figure 1(a), named as primary clot retrieve). If the BGC was blocked by a thrombus and there was no blood flow through the BGC, the BGC was kept inflated and continuous suction was applied via a syringe (Figure 1(b)). Then, the microcatheter together with stent was advanced directly into the site, which was close to the distal tip of the BGC (Figure 1(c)), and unsheathed at the site. The stent together with the delivery microcatheter was then retrieved under continuous suction (Figure 1(d), called rescue clot retrieval). Steps C and D were repeated (i.e., the repeat thrombectomy with a retrieval stent (RTRS) technique) with continuous inflation of the balloon until there was blood flow through the BGC without a thrombus (Figure 1(e)). Contrast was injected via the BGC to confirm successful reperfusion was achieved; then, the BGC was deflated (Figure 1(e)).

\subsection{Outcome Measures}

2.3.1. Clinical Outcomes. The primary clinical efficacy outcome was the rate of good prognosis at 90 days postprocedure, as defined by an mRS score of 0 to 2 . Safety was evaluated by the incidence of symptomatic intracranial hemorrhage $(\mathrm{sICH})$ and all-cause mortality $\leq 90$ days postprocedure.

2.3.2. Angiographic and Procedural Outcomes. The primary angiographic outcome was the rate of achieving successful reperfusion (eTICI $\geq 2$ b, eTICI $\geq 2 c$, and eTICI 3 ), [14] after a single pass of primary clot retrieval. Achievement of complete reperfusion (eTICI 3) after a single pass of primary clot retrieval no matter how many passes of rescue clot retrieval was adpotted, is called the first-pass effect (FPE) [15].

Secondary outcome measures included the incidence of distal emboli, the incidence of emboli in the anterior cerebral artery (ACA), and the final successful reperfusion rate. Successful reperfusion was defined as an eTICI grade of $2 b, 2 c$, or 3 after endovascular treatment. The PRT was defined as the time from puncture to achieving eTICI $\geq 2$ $\mathrm{b}$ or procedure completion.

2.4. Data Availability. Access to patient records for data collection and analysis was approved by our local medical ethics committee, and informed consent was not obtained because of the retrospective nature of the study. We will share the identified data of participants in our study upon request.

2.5. Statistical Analysis. The data for categorical variables are described in absolute and relative frequencies. The data for continuous variables are given as the median and range or the mean and standard deviation. All statistical analyses were performed with IBM SPSS Statistics 22.0 (IBM, Inc., Armonk, NY).

\section{Results}

From 1 January 2015 to 1 November 2020, there were 426 patients with acute intracranial ICA occlusion, and BGCs were used in 116 patients. The BGC was blocked by a thrombus after the first pass of SR, allowing no blood flow out of the BGC, in 40 patients ( 36 cases with $8 \mathrm{~F}$ Merci, 4 cases with 8 F Flowgate 2), and the RTRS technique was applied in 32 patients. An illustration of the cases is shown in Figure 2.

Baseline characteristics, clinical features, and preprocedural radiological features are shown in Table 1 . There were $12(12 / 32,37.5 \%)$ male patients, the age of onset was $73 \pm 11$ 


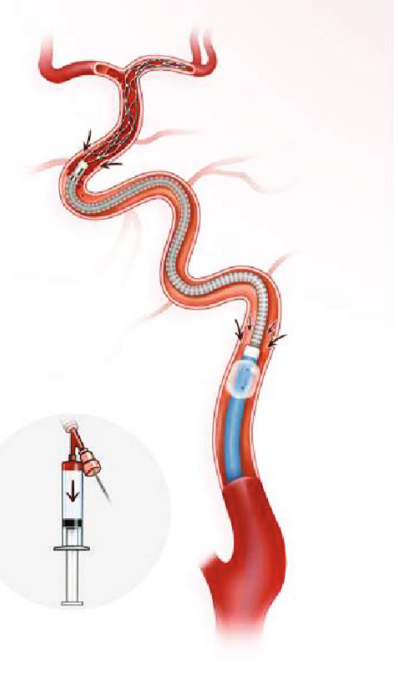

(a)

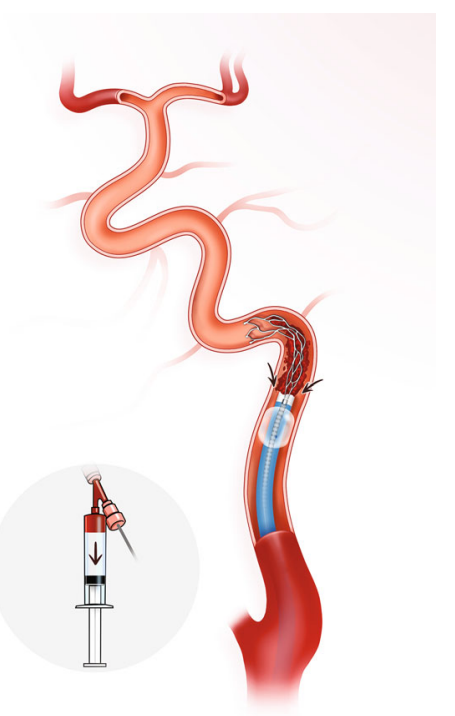

(d)

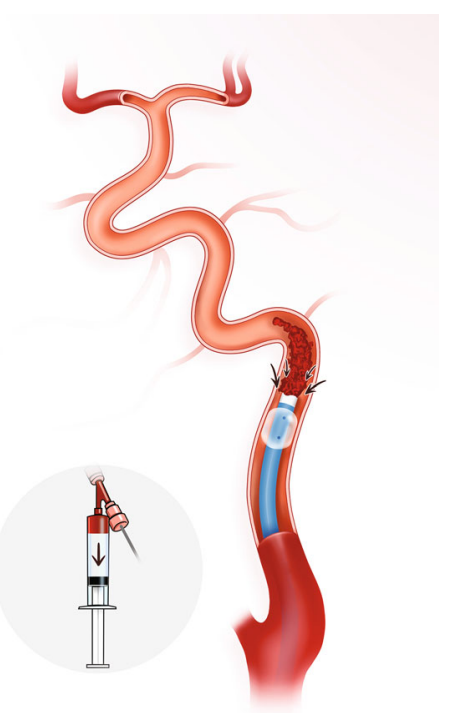

(b)

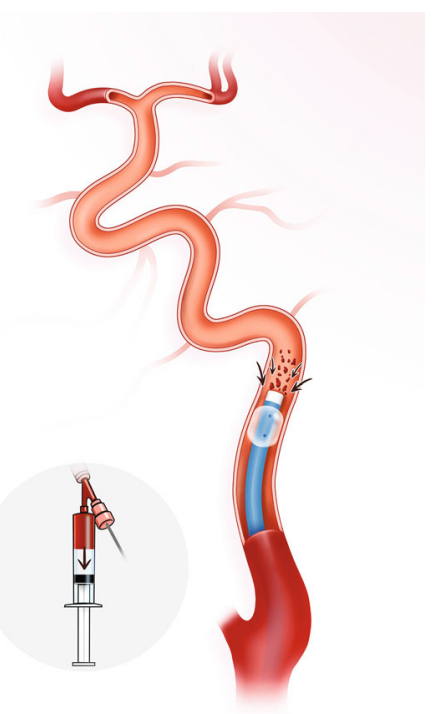

(e)

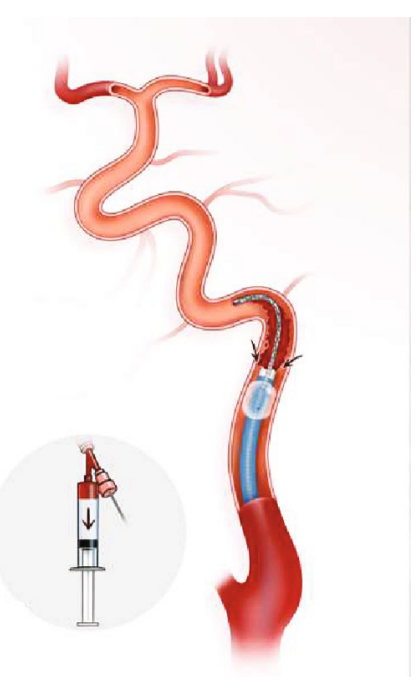

(c)

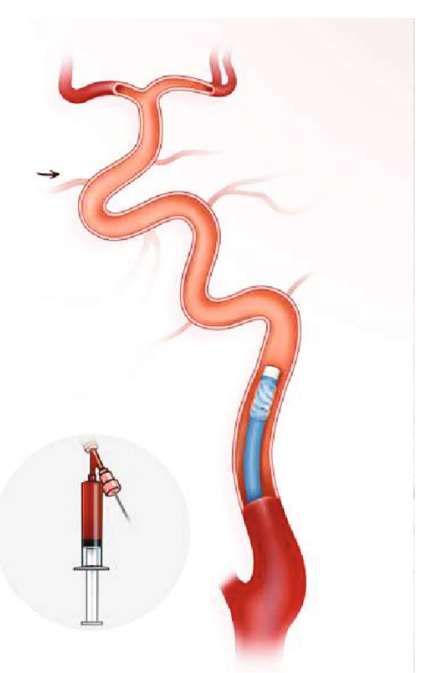

(f)

FIGURE 1: Illustration of the repeat thrombectomy with a retrieval stent (RTRS) technique with continuous proximal flow arrest by a balloon guide catheter for acute intracranial ICA occlusion. (a) DSA shows ICA terminus occlusion involving the MCA and ACA. The retrieval stent is unsheathed at the occlusion site. After at least 3 minutes, the balloon is inflated to arrest the anterograde flow from the ICA, and then the fully deployed Solitaire stent is partially resheathed. Together with the delivery microcatheter and distal access catheter, the stent is gently pulled back under continuous suction achieved with a syringe. This procedure is called primary clot retrieval. (b) The BGC was blocked by a thrombus, and there was no blood flow through the BGC. The BGC was kept inflated, and continuous suction was achieved via a syringe. (c) The microcatheter and stent were advanced together directly into the site, which was close to the distal tip of the BGC. (d) The stent is unsheathed at the site close to the distal tip of the BGC, and then the stent together with the delivery microcatheter is retrieved back under continuous suction. This procedure is called rescue clot retrieval. (e) Blood flow without a thrombus comes out of BGC, and gentle injection contrast via the BGC was performed to confirm successful reperfusion. (f) The BGC was deflated. ICA: internal carotid artery; DSA: digital subtraction angiography; BGC: balloon guide catheter.

years, and the mean admission NIHSS score was 19. Regarding vascular risk factors, $23 / 32$ (71.9\%) patients had hypertension, 6/32 (18.8\%) had diabetes mellitus, 3/32 (9.4\%) had hyperlipidemia, $21 / 32(65.6 \%)$ had atrial fibrillation, and $7 / 32(21.9 \%)$ were smokers.

Procedure details and radiological and clinical outcomes are shown in Table 2. Regarding procedural details, all patients underwent one pass of primary clot retrieval, the median number of rescue clot retrieval passes was 1 (range from 1to 8), the mean PRT was $61.2 \pm 24.0$ minutes, the median PRT was 46 minutes, and the range was from 22 minutes to 142 minutes. After one primary clot retrieval pass, eTICI $\geq 2 b$ reperfusion was achieved in all patients, eTICI $\geq 2$ c reperfusion was achieved in $30 / 32(93.8 \%)$ patients, eTICI 3 reperfusion was achieved in $27 / 32$ $(84.4 \%)$ patients, distal emboli occurred in $2 / 32(6.3 \%)$ 


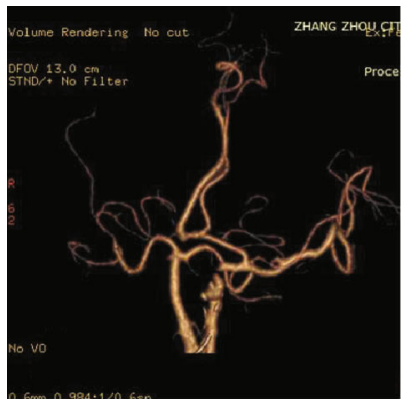

(a)

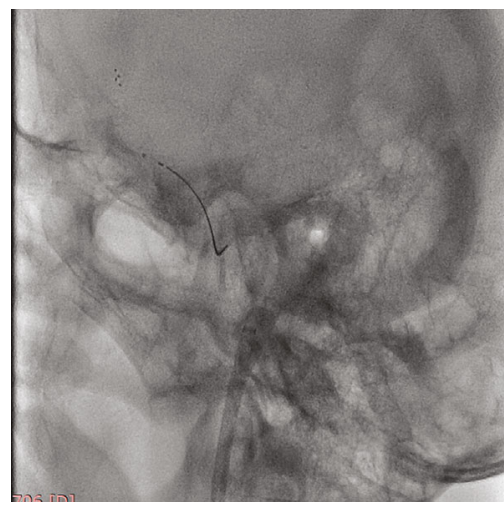

(c)

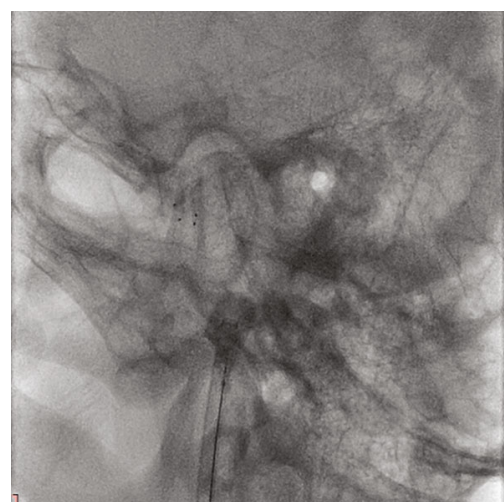

(e)

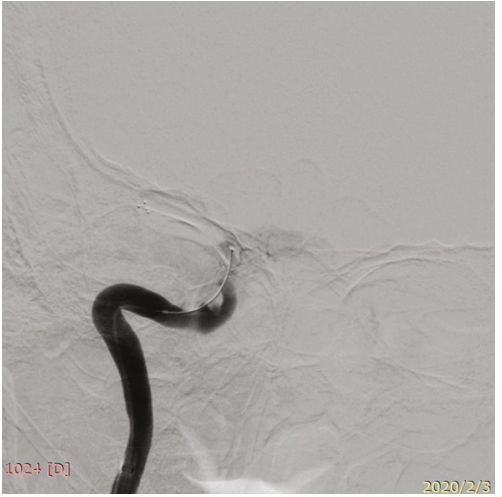

(b)

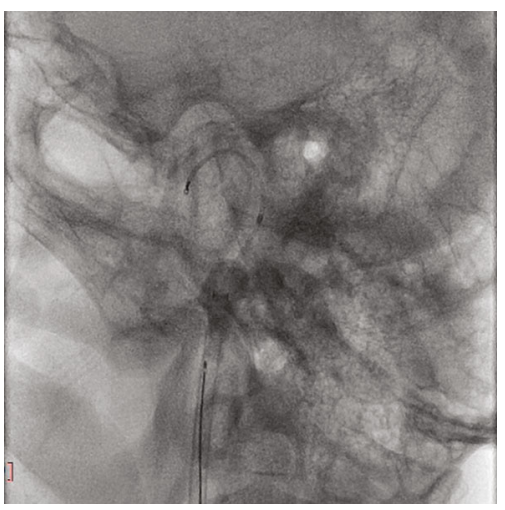

(d)

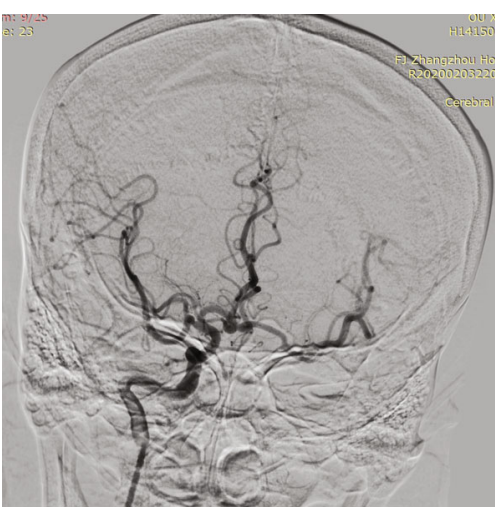

(f)

FIgURE 2: An elderly presented with left hemiparesis and a history of atrial fibrillation postcardiac valve replacement. The patient regularly took warfarin, and admission NIHSS was 18. (a) Computed tomography angiography (CTA) shows total occlusion of the right ICA occlusion and the anterior communicating artery open; the right anterior cerebral artery is supplied by the left ICA. (b) A Solitaire 6-30 $\mathrm{mm}$ stent was unsheathed at the occlusion site (shown as a black arrow). DSA showed ICA intracranial segment occlusion with a large clot burden, and the CBS was 3. (c) After at least 3 minutes, the balloon (shown as a black arrow) was inflated to arrest the anterograde flow from the ICA. Then, the fully deployed Solitaire stent was partially resheathed, and together with the delivery microcatheter, it was gently pulled back under continuous suction achieved with a syringe. This procedure is called primary clot retrieval. (d) The BGC remained inflated for there is no blood out of BGC, and continuous suction was applied via a syringe; the microcatheter and stent are advanced together directly into the site, which is close to the distal tip of the BGC. (e) The stent is unsheathed at the site close to the distal tip of the BGC, and then the stent together with the delivery microcatheter is retrieved back under continuous suction. This procedure is called rescue clot retrieval. (f) Rescue clot retrieval is repeated until there is blood flow without a thrombus through the BGC; contrast is gently injected via the BGC to confirm that successful reperfusion is achieved. The balloon is deflated, and DSA shows that the right ICA is totally patent and the anterior communicating artery is open; the bilateral MCA and ACA are supplied by the right ICA. NIHSS: NIH stroke scale; CTA: CT angiography; DSA: digital subtraction angiography; ICA: internal carotid artery; BGC: balloon guide catheter; CBS: clot burden score; MCA: middle cerebral artery; ACA: anterior cerebral artery. 
TABLE 1: Baseline characteristics, clinical features, and preprocedural radiological features of the 32 patients who underwent RTRS.

\begin{tabular}{lcc}
\hline Item & & \\
\hline & Male sex, $n(\%)$ & $12(37.5 \%)$ \\
$73 \pm 11$ & $382.0 \pm 325.9$ \\
& Age, mean \pm SD (y) & $21(65.6 \%)$ \\
Clinical features & OPT, mean \pm SD (min) & $11(34.4 \%)$ \\
& TOAST-CE & $19(17,21)$ \\
\hline & TOAST-undetermined: cryptogenic embolism & $23(71.9 \%)$ \\
& Admission NIHSS, IQR & $21(18.8 \%)$ \\
Vascular risk factors & Hypertension, $n(\%)$ & $3(95.6 \%)$ \\
& Diabetes mellitus, $n(\%)$ & $7(21.9 \%)$ \\
\hline Preprocedural radiological features & Atrial fibrillation, $n(\%)$ & $4.5(3,5)$ \\
\hline
\end{tabular}

SD: standard deviation; OPT: onset-to-presentation time; TOAST: Trial of Org 10172 in Acute Stroke Treatment; CE: cardioembolism; NIHSS: NIH stroke scale: IQR: interquartile range; CBS: clot burden score.

TABle 2: Procedure details and radiological and clinical outcomes of the 32 patients who underwent RTRS.

\begin{tabular}{|c|c|c|}
\hline Item & & \\
\hline \multirow{5}{*}{ Procedure detail } & One pass of primary clot retrieval, $n(\%)$ & $32(100 \%)$ \\
\hline & Passes of rescue clot retrieval, IQR & $1(1.3)$ \\
\hline & Passes of rescue clot retrieval, median ( $\min , \max )$ & $1(1.8)$ \\
\hline & $\mathrm{PRT}$, mean $\pm \mathrm{SD}(\min )$ & $59.8 \pm 23.1$ \\
\hline & PRT, median (min, max) (min) & $46(22.142)$ \\
\hline \multirow{7}{*}{ Radiological outcome } & One pass of primary clot retrieval achieves & \\
\hline & $\geq$ eTICI $2 \mathrm{~b}$ reperfusion, $n(\%)$ & $32(100 \%)$ \\
\hline & $\geq$ eTICI $2 c$ reperfusion, $n(\%)$ & $30(93.8 \%)$ \\
\hline & PPE: eTICI3 reperfusion, $n(\%)$ & $27(84.4 \%)$ \\
\hline & Distal emboli, $n(\%)$ & $2(6.3 \%)$ \\
\hline & Emboli to the ACA & $0(0 \%)$ \\
\hline & $\mathrm{sICH}$ & $0(0 \%)$ \\
\hline \multirow{2}{*}{ Clinical outcome } & Good prognosis & $14(43.8 \%)$ \\
\hline & Mortality & $8(25.0 \%)$ \\
\hline
\end{tabular}

IQR: interquartile range; SD: standard deviation; OPT: onset-to-presentation time; eTICI: Extended treatment in Cerebral Infarction (eTICI) score; ACA: anterior cerebral artery; FPE: first-pass effect; sICH: symptomatic intracranial hemorrhage; PRT: puncture-to-reperfusion time; FPE: first-pass effect.

patients, and no cases of emboli to the anterior cerebral artery or sICH occurred.

Regarding clinical outcome, the good prognosis rate was $43.8 \%(14 / 32)$, and the mortality rate was $25.0 \%(8 / 32)$.

\section{Discussion}

RTRS is a modification of the conventional SR thrombectomy technique. The modifications of conventional SR thrombectomy are (1) the use of a BGC with the SR thrombectomy technique, (2) continuous inflation of the balloon when removing the retrieval stent and distal access catheter from the guide catheter, and, most importantly, (3) the inclusion of a procedure for when the BGC is blocked by a thrombus and no blood flows out of the BGC. In RTRS, the balloon is kept inflated, and then repeated thrombectomy with a retrieval stent is performed at the site near the distal tip of the BGC until blood flows out of the BGC. Angiography is performed via the BGC by gentle manual injection of contrast agent to ensure that the thrombus is cleared, and then, the balloon is deflated.

The use of RTRS when the BGC is blocked by a thrombus has three useful advantages. First, it is time saving. The BGC does not need to be retracted out of the sheath and reestablished the route. During the rescue clot retrieval procedure, we did not need to advance a microcatheter with a microwire into the middle cerebral artery. We just advanced the microcatheter together with the stent into the site that was close to the distal tip of the BGC and unsheathed the stent at that site, and such an operation was very quick, 
simple, and convenient. Our study showed that the mean time of PRT was shorter than that in a previous study, which was also about endovascular therapy for intracranial artery occlusion (59 minutes vs. 120 minutes) [1]. Second is the high rate of successful reperfusion. Acute intracranial ICA occlusion, especially carotid T occlusion, is usually associated with high clot burden and low CBS [16], and the successful reperfusion rate ranges from $60.5 \%$ [1] to $86.9 \%$ [17]. In our study, all patients achieved successful reperfusion, and approximately $90.9 \%$ of patients achieved nearly complete successful reperfusion after one primary clot retrieval pass. Third is high FPE occurrence. FPE is associated with significantly higher rates of good clinical outcome $[9,15]$. A previous study showed that FPE was observed in $33 \%$ of patients with intracranial artery occlusion who underwent SR thrombectomy with $8 \mathrm{~F}$ Flowgate 2BGC in which the inner lumen was larger than that of $8 \mathrm{~F}$ Merci. In our study, FPE was observed in $81.8 \%$ of patients. Fourth, there was a low occurrence of distal emboli and emboli into the ACA. Distal emboli are a troublesome event during mechanical thrombectomy and is usually associated with increased mortality and disability rates, regardless of the success of reperfusion [18]. Distal emboli are a controllable event. Acute intracranial ICA occlusion, which is associated with a high clot burden, had a higher rate of distal emboli (15.3\%) than middle cerebral artery (MCA) occlusion (4.8\%) [18]. The use of mechanical thrombectomy (MT) techniques can decrease the incidence of distal emboli. For example, the use of distal access catheters in association with $\mathrm{SR}$, which is common in clinical practice, can decrease the distal emboli incidence from $5 \%$ to $11 \%$ [19]. The use of BGCs can also decrease the incidence of distal emboli [20, 21]. Regarding intracranial ICA occlusion, the incidence of distal emboli is as high as $75 \%$ in patients who underwent MT with conventional guide catheters [20], but the incidence decreases to $0 \%$ when MT is combined with BGCs [20]. In our study, the incidence of distal emboli was $6.3 \%$, with no emboli to the ACA. Regarding cases with distal emboli, the thrombus is very small, so it causes only very remote branches to become occluded, which may minimize the influence on functional outcome.

There are three important technical points of the RTRS technique. The first point is continuous inflation of the balloon when there is no blood flow coming out of the BGC and never performing angiography with contrast during this step because of the risk of moving the thrombus into a distal territory with the injection pressure. Second, repeat thrombectomy with a retrieval stent should not be stopped at the site near the tip of the BGC until blood is flowing out of the BGC and no thrombus is trapped by the stent. Third, when the blood flow from the BGC is not fluent and no thrombus is trapped by the stent, two situations should be considered. One is that the tip of the BGC is completely or partially covered by the vessel wall, and the other is that there is a thrombus in the ICA terminus. Therefore, we needed to partly unsheath the stent at the ICA terminus, deflate the balloon, gently retract the BGC slightly, and then perform angiography via the BGC to check what happened. If complete recanalization is achieved, the procedures terminated; if there is thrombus in the culprit artery, the balloon is reinflated, and the thrombectomy was repeated.

There is one concern about cerebral ischemia caused by continuous proximal blood flow arrest using balloon occlusion, which has also been reported with the TSAT technique [21]. However, we only adopt this technique in patients with intracranial ICA occlusion, so we do not need to worry about this problem because the vessel is initially occluded and there is no blood supply downstream territory. Another concern is the formation of fresh thrombi secondary to continuous proximal blood flow arrest. Therefore, the procedure should be performed as soon as possible, and proper heparinization is also needed during the procedure.

Due to the excellent performance of the RTRS technique on radiological outcomes, the clinical outcome of the patients in our study was acceptable and even better than that of those in a previous study, although the thrombus burden was large in our included cases, which is usually associated with poor clinical outcomes. The clinical outcome is poor in patients with intracranial ICA occlusion $[17,22$, 23], the rate of good outcomes ranges from $21.4 \%$ [1] to $40 \%$ [17], and the mortality rate ranges from $29.3 \%$ [22] to $55.2 \%$ [1]. In our study, the incidence of good outcomes was $43.8 \%$, and the mortality rate was $25.0 \%$.

This study is limited by being a single-center study. Despite this limitation, our study is the first series with relatively numerous cases that attempted to examine an endovascular strategy in the setting of the BGC being blocked by a thrombus. Second, the 8 F Flowgate2 BGC inner lumen (0.084 inches) of which is larger than that of the $8 \mathrm{~F}$ Merci (0.078 inches), was not used in all cases in our study. However, we only present the technique to address the circumstance of the BGC being blocked by a thrombus, which could also occurred in cases with $8 \mathrm{~F}$ Flowgate 2 BGCs or even those with larger inner lumens (0.090 inches) [24], so this usage of this technique is not influenced.

\section{Conclusion}

RTRS with proximal flow arrest using a BGC is effective for the treatment of acute ischemic stroke due to acute intracranial ICA occlusion. This approach has allowed us to achieve excellent clinical and angiographic outcomes and shorten the PRT time, and it may reduce the incidence of distal emboli in acute ischemic thrombectomy. This technique should be considered in cases of acute intracranial ICA occlusion when the BGC is blocked by a thrombus during the procedure. However, our findings should be confirmed by future multicenter, large-sample studies.

\section{Data Availability}

We declare that we can make data available on request through institutional review board once it is needed.

\section{Conflicts of Interest}

The authors declare that they have no conflicts of interest. 


\section{Acknowledgments}

This study was supported by the National Health Commission Capacity Building and Continuing Education Center (grant number GWJJ2021100203). We thank the team of American Journal Expert for editing the English text of a draft of this manuscript (https://secure.aje.com).

\section{References}

[1] D. S. Liebeskind, A. C. Flint, R. F. Budzik et al., "Carotid I's, L's and T's: collaterals shape the outcome of intracranial carotid occlusion in acute ischemic stroke," Journal of NeuroInterventional Surgery, vol. 7, no. 6, pp. 402-407, 2015.

[2] O. A. Berkhemer, P. S. S. Fransen, D. Beumer et al., "A randomized trial of intraarterial treatment for acute ischemic stroke," The New England Journal of Medicine, vol. 372, no. 1, pp. 11-20, 2015.

[3] B. C. V. Campbell, P. J. Mitchell, T. J. Kleinig et al., "Endovascular therapy for ischemic stroke with perfusion-imaging selection," The New England Journal of Medicine, vol. 372, no. 11, pp. 1009-1018, 2015.

[4] M. Goyal, A. M. Demchuk, B. K. Menon et al., "Randomized assessment of rapid endovascular treatment of ischemic stroke," The New England Journal of Medicine, vol. 372, no. 11, pp. 1019-1030, 2015.

[5] J. L. Saver, M. Goyal, A. Bonafe et al., "Stent-retriever thrombectomy after intravenous t-PA vs. t-PA alone in stroke," The New England Journal of Medicine, vol. 372, no. 24, pp. 2285-2295, 2015.

[6] T. G. Jovin, A. Chamorro, E. Cobo et al., "Thrombectomy within 8 hours after symptom onset in ischemic stroke," The New England Journal of Medicine, vol. 372, no. 24, pp. 22962306, 2015.

[7] R. G. Nogueira, A. P. Jadhav, D. C. Haussen et al., “Thrombectomy 6 to 24 hours after stroke with a mismatch between deficit and infarct," The New England Journal of Medicine, vol. 378, no. 1, article NEJMoa1706442, pp. 11-21, 2018.

[8] G. W. Albers, M. P. Marks, S. Kemp et al., "Thrombectomy for stroke at 6 to 16 hours with selection by perfusion imaging," The New England Journal of Medicine, vol. 378, no. 8, pp. 708-718, 2018.

[9] O. Nikoubashman, S. Dekeyzer, A. Riabikin et al., "True firstpass Effect," Stroke, vol. 50, no. 8, pp. 2140-2146, 2019.

[10] M. Mazighi, S. A. Chaudhry, M. Ribo et al., "Impact of onsetto-reperfusion time on stroke Mortality," Circulation, vol. 127, no. 19, pp. 1980-1985, 2013.

[11] A. Todo, A. Minaeian, R. Sahni, and K. H. Chao, "Incidence and outcome of procedural distal emboli using the penumbra thrombectomy for acute stroke," Journal of NeuroInterventional Surgery, vol. 5, no. 2, pp. 135-138, 2013.

[12] O. Nikoubashman, D. Wischer, H. M. Hennemann et al., "Balloon-guide catheters are needed for effective flow reversal during mechanical thrombectomy," American Journal of Neuroradiology, vol. 39, no. 11, pp. 2077-2081, 2018.

[13] W. Brinjikji, R. M. Starke, M. H. Murad et al., "Impact of balloon guide catheter on technical and clinical outcomes: a systematic review and meta-analysis," Journal of NeuroInterventional Surgery, vol. 10, no. 4, pp. 335-339, 2018.

[14] D. S. Liebeskind, S. Bracard, F. Guillemin et al., "ETICI reperfusion: defining success in endovascular stroke therapy," Jour- nal of NeuroInterventional Surgery, vol. 11, no. 5, pp. 433-438, 2019.

[15] O. O. Zaidat, A. C. Castonguay, I. Linfante et al., "First pass Effect," Stroke, vol. 49, no. 3, pp. 660-666, 2018.

[16] I. Derraz, R. Bourcier, M. Soudant et al., "Does clot burden score on baseline T2*-MRI impact clinical outcome in acute ischemic stroke treated with mechanical thrombectomy?," Journal of Stroke, vol. 21, no. 1, pp. 91-100, 2019.

[17] T. Ota, K. Shigeta, T. Amano, and J. Kaneko, "Endovascular treatment for acute carotid $\mathrm{T}$ and carotid non-T occlusion: a retrospective multicentre study of 81 patients," Interventional Neuroradiology, vol. 25, no. 5, pp. 497-501, 2019.

[18] V. Chalumeau, R. Blanc, H. Redjem et al., "Anterior cerebral artery embolism during thrombectomy increases disability and mortality," Journal of NeuroInterventional Surgery, vol. 10, no. 11, pp. 1057-1062, 2018.

[19] R. A. McTaggart, E. L. Tung, S. Yaghi et al., "Continuous aspiration prior to intracranial vascular embolectomy (CAPTIVE): a technique which improves outcomes," Journal of NeuroInterventional Surgery, vol. 9, no. 12, pp. 1154-1159, 2017.

[20] S. H. Kim, J. H. Choi, M. J. Kang et al., "Efficacy of combining proximal balloon guiding catheter and distal access catheter in thrombectomy with stent retriever for anterior circulation ischemic stroke," Journal of Korean Neurosurgical Association, vol. 62, no. 4, pp. 405-413, 2019.

[21] H. Matsumoto, H. Nishiyama, Y. Tetsuo, H. Takemoto, and N. Nakao, "Initial clinical experience using the two-stage aspiration technique (TSAT) with proximal flow arrest by a balloon guiding catheter for acute ischemic stroke of the anterior circulation," Journal of NeuroInterventional Surgery, vol. 9, no. 12, pp. 1160-1165, 2017.

[22] D. Frahm, S. Wunderlich, M. I. Schubert, H. Poppert, J. F. Kleine, and S. Prothmann, "Mechanical thrombectomy in acute occlusion of the carotid-T: a retrospective single centre study in 51 patients," Clinical Neuroradiology, vol. 26, no. 1, pp. 23-29, 2016.

[23] G. B. Bradac, F. Venturi, G. Bosco et al., "Acute occlusion of the distal internal carotid Artery," Clinical Neuroradiology, vol. 30, no. 1, pp. 67-76, 2020.

[24] A. Okamura, K. Kuroki, K. Shinagawa, and N. Yamada, "Simple aspiration with balloon catheter technique (simple $\mathrm{ABC}$ technique) against proximal internal carotid artery occlusion in cases of cardiogenic cerebral embolism," Interventional Neuroradiology, vol. 24, no. 3, pp. 317-321, 2018. 